\title{
From Bioconjugation to Self-Assembly in Nanobiotechnology: Quantum Dots Trapped and Stabilized by Toroid Protein Yoctowells**
}

\author{
By Andreas Schreiber, Ying Yuan, Matthias C. Huber, Ralf Thomann, Andreas Ziegler, \\ Helmut Cölfen, Jörn Dengiel, Michael Krüger and Stefan M. Schiller*
}

Conjugation of quantum dots (QDs) with proteins is applicable for biosensing and imaging in biomedical applications but also for the confined assembly of various nanostructures. Here, the conjugation of CdSe QDs ranging from 2.5 up to $9.5 \mathrm{~nm}$ with Hcp1, a yoctowell forming hexameric core protein of Pseudomonas aeruginosa, is described. Defined structures ranging from single QDs trapped within the donut-shaped hexameric protein cavity over "globular" multi-toroid architectures up to photonic wires of $850 \mathrm{~nm}$ in length and $9 \mathrm{~nm}$ in width are self-assembled while preserving the native hexameric structure of the protein conserving the photoluminescence intensity of the QDs in aqueous solution for more than 3 month.

Defined molecular structures composed of organic/biological and inorganic materials as well as their functional assemblies are of great importance in the developing field of nanobiotechnology. They show a great potential in designing new materials with advanced photonic, electronic, and magnetic properties for applications in science, technology, and medicine. A major advantage of bio-based building blocks is due to the fact that they tend to have a multifunctional character. Multifunctionality
[*] A. Schreiber, M. C. Huber, Dr. S. M. Schiller Freiburg Institute for Advanced Studies (FRIAS) School of Soft Matter Research, University of Freiburg Albertstr. 19, 79104 Freiburg, Germany E-mail: stefan.schiller@frias.uni-freiburg.de A. Schreiber, M. C. Huber, R. Thomann, Dr. S. M. Schiller Institute for Macromolecular Chemistry University of Freiburg, Stefan-Meier-Str. 31 79104 Freiburg, Germany

A. Schreiber, Dr. S. M. Schiller

Faculty of Biology, Schaenzlestrasse 1

D-79104 Freiburg, Germany

Y. Yuan, R. Thomann, M. Krüger

Freiburg Materials Research Centre

University of Freiburg, Stefan-Meier-Str. 21

79104 Freiburg, Germany

A. Ziegler

University of Ulm

Albert Einstein Allee 11, 89069 Ulm, Germany

H. Cölfen

Department of Chemistry, University of Konstanz

Universitätsstraße 10, Box 714

D-78457 Konstanz, Germany

\author{
J. Dengjel \\ Freiburg Institute for Advanced Studies \\ School of Life Science-LIFENET, University of Freiburg \\ Albertstr. 19, 79104 Freiburg, Germany \\ J. Dengjel \\ Center for Biological Systems Analysis, ZBSA \\ University of Freiburg, Habsburgerstr. 49 \\ 79104 Freiburg, Germany \\ J. Dengjel, Dr. S. M. Schiller \\ BIOSS Centre for Biological Signalling Studies \\ University of Freiburg, Hebelstrasse 25 \\ D-79104 Freiburg, Germany
}

[**] The authors would like to thank Prof. Joseph D. Mougous for the Hcp1Q54C containing plasmid. We are grateful to the Freiburg Institute for Advanced Studies (FRIAS), the Deutsche Forschungs Gemeinschaft DFG (german science foundation, EXC 294) BIOSS Centre for Biological Signalling Studies, the "Kompetenznetz funktionelle Nanostrukturen" (competence network of functional nanostructures, KFN), the BadenWürttemberg Stiftung, the Ministerium für Wissenschaft, Forschung und Kunst des Landes Baden-Württemberg (Ministry of Science, Research and the Arts (MWK) Baden-Württemberg) and the Rectorate of the University of Freiburg for support. Supporting Information is available online from Wiley Online Library or from the author. 
combined with precise molecular structures (sequence and three-dimensional shape) is a prerequisite to design complex functions and selective interactions in complex systems. We therefore designed complex protein-building blocks with defined shapes in the range of $10 \mathrm{~nm}$ modified with specific functionalities oriented at precise 3D-positions. Possible applications of photonic systems in bionanotechnology may span the range from light converting systems to fluorescent bioconjugates such as bio-functionalized quantum dots (QDs).

In order to use the great potential of QDs in biomedical applications they need to be bio-functionalized, water soluble, prevented from photoluminescence quenching in aqueous solution and non-toxic. Different methods can be applied to access QDs that are stable in aqueous solution. Examples include polymeric coatings introducing hydrophilic or electrical charges and signal sequences. ${ }^{[1]}$ Moreover, nanocrystals can be made hydrophilic by surface coatings carrying different functional groups, exhibiting reactivity toward the nanocrystal at one side and rendering them water soluble on the other side. Often, thiol groups are used to bind the QDs, whereas carboxylic groups facilitate the solubility in aqueous solution. ${ }^{[2,3]}$ Besides capping CdSe QD with different organic ligands or polymers, another evolving method is bioconjugation which also renders QDs water soluble. ${ }^{[4]}$ Toxicity of CdSe toward cells also depends on surface coatings, which affect the amount of $\mathrm{Cd}^{2+}$ released to the cell medium. ${ }^{[5,6]}$ To prevent the harmful $\mathrm{Cd}^{2+}$ liberation appropriate coatings like polyethylene glycol or a ZnS shell in combination with BSA proofed to reduce cytotoxicity tremendously. ${ }^{[6,7]}$ But also CdSe QDs functionalized with elastin like proteins displayed no toxicity toward cells. ${ }^{[8]}$ Bio functionalization of QDs with proteins, peptides or DNA for various applications has become increasingly important over the past decade. ${ }^{[9-12]} \mathrm{A}$ few demands need to be full filled for bioconjugation. ${ }^{[13]}$ The bound nanoparticle should not impair the nature of the protein and and its photoluminescence should not be quenched by the bio molecule. Moreover, attaching a defined number of biomolecules is desirable to control the number of linkage sites to the QD. Furthermore, bio labeling applications in cell biology can be improved via protein or peptide modification and ought to facilitate membrane permeability.

\section{Experimental}

Synthesized CdSe QDs of different sizes and the Hcp1 pore protein from Pseudomonas aeruginosa were used for defined bioconjugation experiments presented here. Hcp1 is a ring shaped homo-hexameric pore protein, which was first described by Mougous et al. ${ }^{[14]}$ The $112 \mathrm{kDa}$ protein has an outer diameter of $9.0 \mathrm{~nm}$, an inner diameter of $4.0 \mathrm{~nm}$ and a height of $4.4 \mathrm{~nm}$ (Figure 1). Each subunit contains one genetically encoded cysteine at position Q54C and one C-terminal His6-tag (in the paper referred to as Hcp1).

Hcp1 was expressed in large scale in Escherichia coli BL21 stain, grown in a $7 \mathrm{~L}$ fermenter and purified via a Ni-NTA column with a yield of $0.15 \mathrm{mg} \cdot \mathrm{ml}^{-1}$ cell culture.

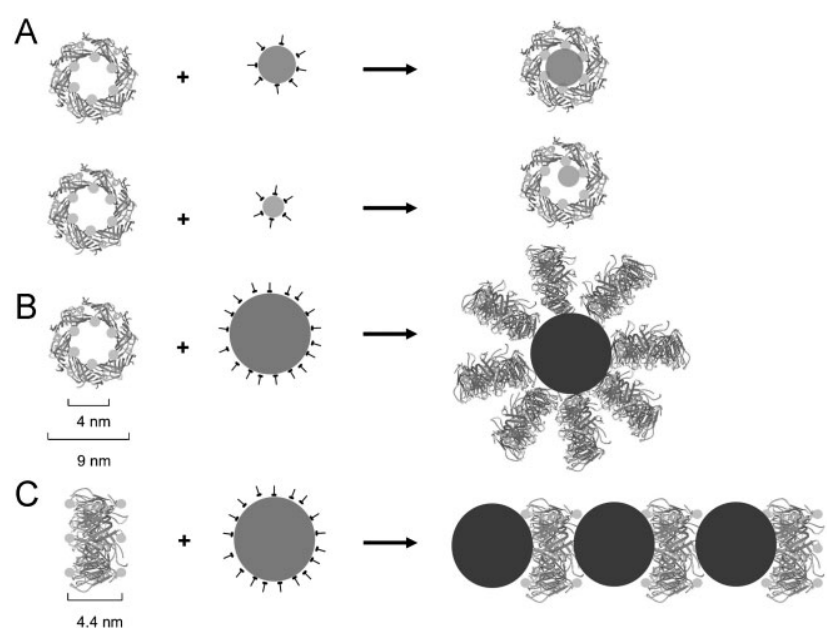

Fig. 1. (A) Interaction scheme of Hcp1 and $4.5 \mathrm{~nm} Q D s$ (red), $2.5 \mathrm{~nm} Q D s$ (green) and $9.5 \mathrm{~nm} Q D s$ (brown) with an HDA ligand trapped via thiol-QD interactions inside the cavity. (B) Model of Hcp1 and $9.5 \mathrm{~nm} Q D s$ (brown) forming toroid like structures. (C) Assembly model of Hcp1Cys3 and $9.5 \mathrm{~nm}$ QDs to biohybrid fibers.

During a routine preparation, CdSe QDs with diameters $<7 \mathrm{~nm}$ were synthesized at $300^{\circ} \mathrm{C}$ from Cd-stearate and trioctylphosphine-selenide (TOPSe) precursors in an organic matrix consisting of trioctylphosphine-oxide (TOPO) and hexadecylamine (HDA) using a hot injection method described in detail recently. ${ }^{[15]}$ A small modification was made to the $300^{\circ} \mathrm{C}$ hot injection method for obtaining the $9.5 \mathrm{~nm}$ sized QDs [see Supporting Information]. After synthesis photoluminescence (PL), absorption and concentration were assessed according to the method described by $\mathrm{Yu}$ et al. ${ }^{[16]}$ During the phase transfer reaction, the differently sized CdSe QDs (2.5, 3.5, 4.5, and $9.5 \mathrm{~nm}$ in diameter) in various organic solvents incubated with the Hcp1 containing buffer solution by stirring or shaking at room temperature. After the reaction, the lower QD-Hcp1 conjugate containing buffer phase was separated very carefully from the upper toluene phase. PL was recorded for the bioconjugates containing water phase and the unreacted QDs in the organic phase. To maximize the yield of PL and bioconjugate stability, various experimental conditions were tested, such as: different QD-ligands (pyridine, HDA), different organic solvents (toluene, DMSO, and chloroform), and varying reaction times. In order to control the assembly process of the bioconjugate and the type of nanostructures yielded, different QD sizes and different Hcp1 variants were tested. The QD-Hcp1 conjugates and the formed structures were characterized via PL, transmission electron microscopy (TEM), fluorescence microscopy, circular dichroism (CD)-spectroscopy, and intrinsic tryptophan fluorescence. Size distribution was assessed by sodium dodecyl sulfatepolyacrylamide gel electrophoresis (SDS-PAGE)-and native gel electrophoresis. For detailed information please see the Supporting Information.

For the conjugation experiments two different mutants were deployed: Hcp1Q54C (Hcp1) (Figure 1A) and 
Hcp1Q54C-G91C-N159C (referred to as Hcp1Cys3) (Figure 1C). Cysteins introduced at the inner cavity surface (Figure 1A Q54C marked as yellow dots) facilitated the specific QD-binding and incorporation into the toroid protein. Due to the strong interaction between semi-conductor QDs and thiols ${ }^{[17,18]}$ QDs bind preferentially to the thiols placed at the center of the toroid hexameric Hcp1. To guide the directional assembly of bioconjugates, a new variant was designed. Additional cysteines were introduced at the upper and lower rim of the toroid (G91C and N159C, marked as yellow dots in Figure 1B) to create new sites for a potential QD attachment and new structures (Figure 1C).

\section{Results and Discussion}

The phase transfer reaction was conducted in different solvent mixtures. While shaking or stirring the organic solvent with the buffer phase, the QDs interact with the Hcp1 protein and transfer from the organic phase into the aqueous phase. Differently structured bioconjugates between Hcp1 and the QD are formed while a partial ligand exchange (e.g., HDA $\rightarrow$ Hcp1) can be assumed. The PL of the differently sized Hcp1-QD conjugates is detectable in the water phase at the appropriate wavelength. After conjugation, no shift between the maximal PL of conjugates in the water and the QDs in the toluene phase is observed as reported elsewhere for some conjugates. ${ }^{[17]}$ For both, the toluene and the buffer phase, the PL is constant for the $2.5 \mathrm{~nm}$ QD at $530 \mathrm{~nm}$ (not shown), $3.5 \mathrm{~nm}$ at $581 \mathrm{~nm}, 4.5 \mathrm{~nm}$ at $610 \mathrm{~nm}$, and the $9.5 \mathrm{~nm}$ QD at $671 \mathrm{~nm}$ (Figure 2). For all used sizes in toluene and buffer as well, the full-width at half-maximum of the PL peak is below $45 \mathrm{~nm}$, which indicates a narrow size distribution of $\pm 0.5 \mathrm{~nm}$ for the QDs before and after binding to Hcp1. While shaking the phase transfer reaction at $800 \mathrm{rpm}$ the PL was measured after 2, 15, 20, and $44 \mathrm{~h}$. The maximal PL intensity of the conjugates was reached after $20 \mathrm{~h}$ reaction time. Especially for the $9.5 \mathrm{~nm}$ QD it was observed that QDs in the toluene phase seemed to be quenched after the reaction which could

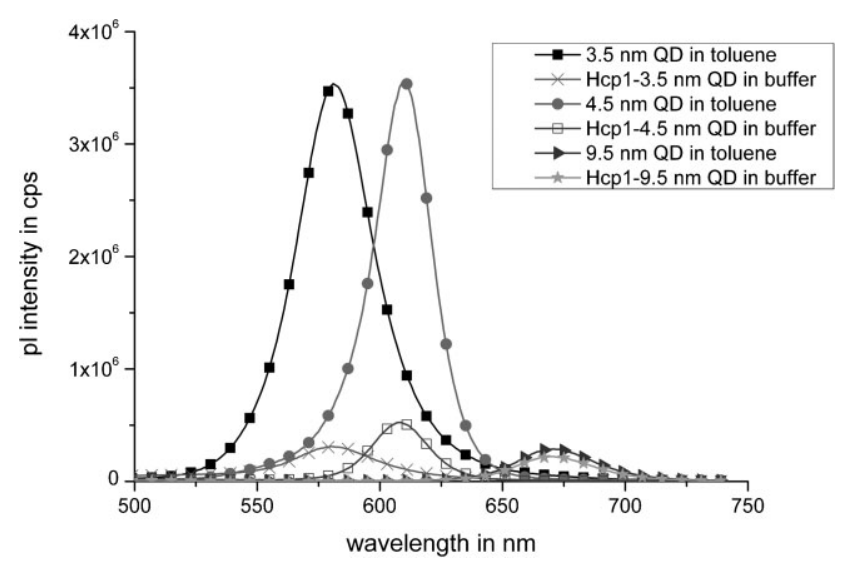

Fig. 2. PL intensity in cps (counts per s) of different sized Hcp1-QD conjugates in buffer and toluene after $20 \mathrm{~h}$ of stirring of the toluene/buffer phase. be due to aggregation of the $9.5 \mathrm{~nm}$ QDs. The concentration of the QDs in the protein buffer phase could not be assessed directly via absorption measurements because of blurring. Instead, the concentration in toluene before and after the reaction was determined and the difference was calculated (for details see Supporting Information). As CdSe QDs are not stable in all organic solvents the best compatibility had to be found for both the organic phase and the buffer phase. After solvent optimization (for details see Supporting Information) toluene and $50 \times 10^{-3} \mathrm{M}$ phosphate buffered saline (PBS) buffer with $250 \times 10^{-3} \mathrm{M} \mathrm{NaCl}$ was found to be the best solvent combination, hence yielding the highest PL for pyridine and HDA capped QD-Hcp1 conjugates of the buffer phase after phase separation (see Figure S1A in the Supporting Information). Therefore, toluene and PBS buffer were used as standard phase transfer reaction solvents. As DMSO is fully miscible with water and provides a better biocompatibility, DMSO was also tested as organic solvent for bioconjugation. After the reaction, $\mathrm{PL}$ intensity was recorded for all conjugates in DMSO $/ \mathrm{H}_{2} \mathrm{O}$ and analyzed by TEM. The control samples which did contain just buffer and QDs (no Hcp1) show a decreased PL after mixing for $20 \mathrm{~h}$ and no PL after 3 days incubation at $4{ }^{\circ} \mathrm{C}$ whereas the Hcp1-QD conjugates still exhibit strong PL after several weeks (see Figure S1B in the Supporting Information). This indicates an enhancement of the QD emission through bioconjugation. As a result of the bioconjugation between QDs and Hcp1, the QDs are shielded, stabilized, and their PL is not quenched in aqueous solution.

Apart from the solvent, QD ligands also affect the observed PL of the bioconjugates. The donut shaped Hcp1 binds the QDs via ligand exchange by replacing or partly replacing the former QD-ligand. Pyridine and HDA were compared in order to find the best exchangeable ligand. After conjugation, the PL signal of the $4.5 \mathrm{~nm}$ pyridine QD is about threefold stronger than that of the HDA bound QDs (see Figure S2 in the Supporting Information). Due to the weaker interaction of the pyridine nitrogen with QDs, it exchanges more efficiently compared to primary amines such as HDA and provides a better surface accessibility for the interaction with the Hcp1 protein. ${ }^{[19,20]}$ In single-sized exposure to Hcp1, the $4.5 \mathrm{~nm}$ QDs show the best PL in the buffer phase after the exchange reaction. The PL of the Hcp1-4.5 nm QD conjugates is threefold higher than for the 9.5 and $2.5 \mathrm{~nm}$ QDs at the same concentration (Figure 3). According to the crystal structure of Hcp $1,{ }^{[14]}$ the inner cavity of the protein is about $4.0 \mathrm{~nm}$. Proving the assumption of the crystal structure assessment the $4.5 \mathrm{~nm}( \pm 0.5 \mathrm{~nm})$ QD fits tightly into the cavity (Figure 5). Further analyzing the crystal structure of Hcp1 (Figure S3 in the Supporting Information) reveals a cavity with dominating negatively charged hydrophilic residues allowing thiol-Cd ${ }^{2+}$ binding and preventing hydrophobic QD-Hcp1 interactions. Hence, inside the cavity the QDs are stabilized by multiple thiol surface interactions (six thiols per hexamer) increasing the conjugation stability. ${ }^{[21]}$ The surrounding protein shell protects the QD from the water molecules and enhances their PL intensity. Usually, the thiol-QD interaction leads to 


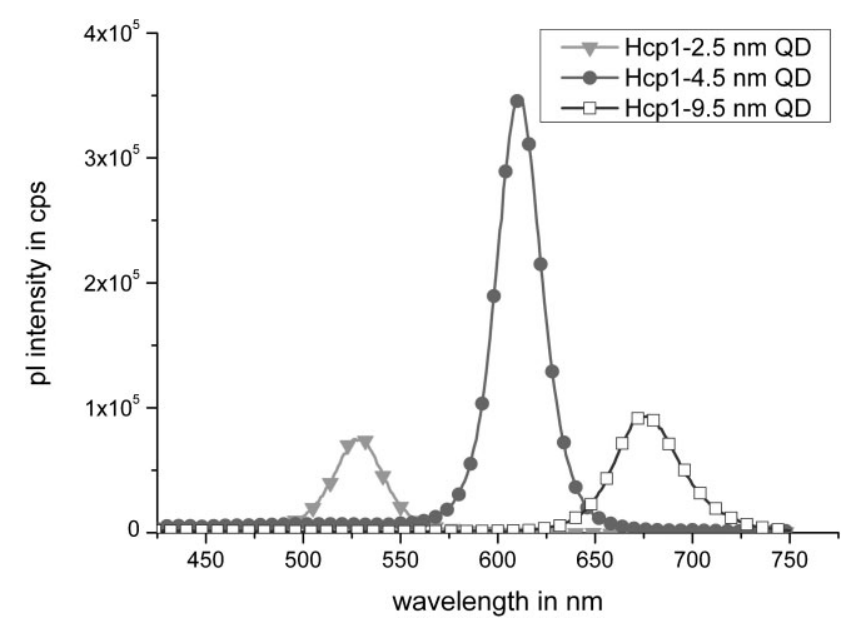

Fig. 3. PL intensity of different sized Hcp1-QD conjugates in buffer after $20 \mathrm{~h}$ of stirring of the toluene/buffer phase.

quenching ${ }^{[20]}$ whereas in this case the complexing thiols and the protection benefits of the protein shell prevails the thiol quenching of the Hcp1-QDs conjugates in buffer. If Hcp1 is exposed to three different sizes of QDs in mixture (2.5, 4.5, and $9.5 \mathrm{~nm}$ ), decreased PL intensity at 530 and $610 \mathrm{~nm}$ (corresponds to 2.5 and $4.5 \mathrm{~nm}$ QDs) and increased PL at $671 \mathrm{~nm}$ (9.5 nm QDs) is observed (Figure 4). The sum of the PL intensity loss of the two smaller sized QDs conjugates is about the same value as the PL intensity increase of the $9.5 \mathrm{~nm}$ QD in mixture (Figure 4). The enhanced PL at $671 \mathrm{~nm}$ intensity is due to the energy transfer (FRET) of the smaller to larger QDs. The reason for the energy transfer is, on the one hand, the wide excitable spectral range of QDs and, on the other hand, the close spatial proximity of less than $10 \mathrm{~nm}$ of the assembled QD-protein conjugates.

The differently sized $(2.5-9.5 \mathrm{~nm})$ QDs are all bound by Hcp1. Thus, the conjugates are not only formed by those QDs that fit into the $4 \mathrm{~nm}$ wide cavity and interact with the thiols at

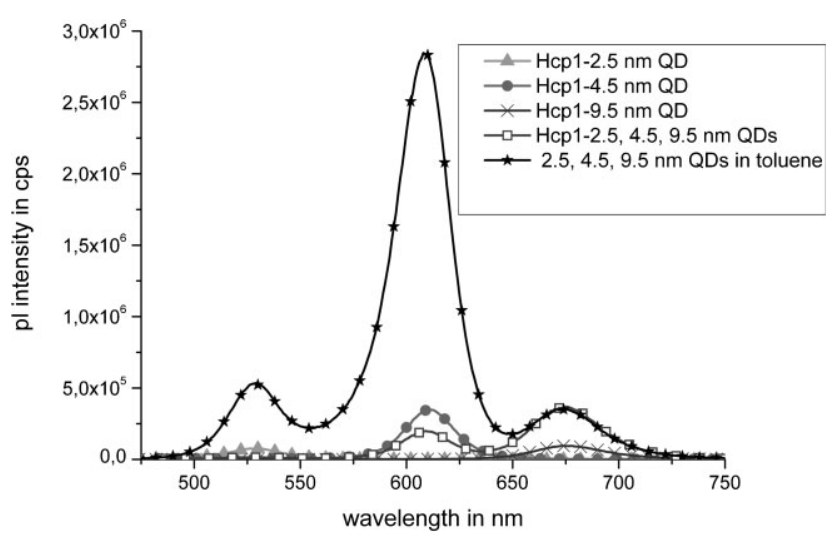

Fig. 4. PL intensity of the single Hcp1-QD conjugates of different sizes in buffer and the $Q D$-mix in toluene after $20 \mathrm{~h}$ of stirring of the toluene/buffer phase.

the inside. Hence, various binding motifs and structures formed are accessible. According to the literature, different motifs for protein-QD binding are reported ${ }^{[9]}$ e.g., via His-tag, ${ }^{[22-24]}$ hydrophobic interactions, carboxy goups, or thiol binding. ${ }^{[21,25]}$ To further investigate the binding scheme and the bioconjugate structures, a set of three further experiments was conducted: (A) column purification of the Hcp1-QD conjugates; (B) fluorescence- and TEM-imaging of the formed structures, SDS-PAGE, (C) native gel electrophoresis and long term PL intensity was measured. (A) It was tested whether the 4.5 and $9.5 \mathrm{~nm}$ QD-Hcp1 conjugates still show their native binding behavior via His tag to the Ni-NTA resin. Therefore, Hcp1 and QDs of different sizes alone and in mixture were linked in DMSO/buffer. The Hcp1-9.5 QD conjugates were separated from the non-conjugated QDs via a standard Ni-NTA column as the usual protein purification procedure (for details see Supporting Information). After applying the samples to the columns, flow through, washing-, and elution steps were collected and analyzed via fluoroscope, SDS-PAGE, and TEM. Flow through and washing steps did
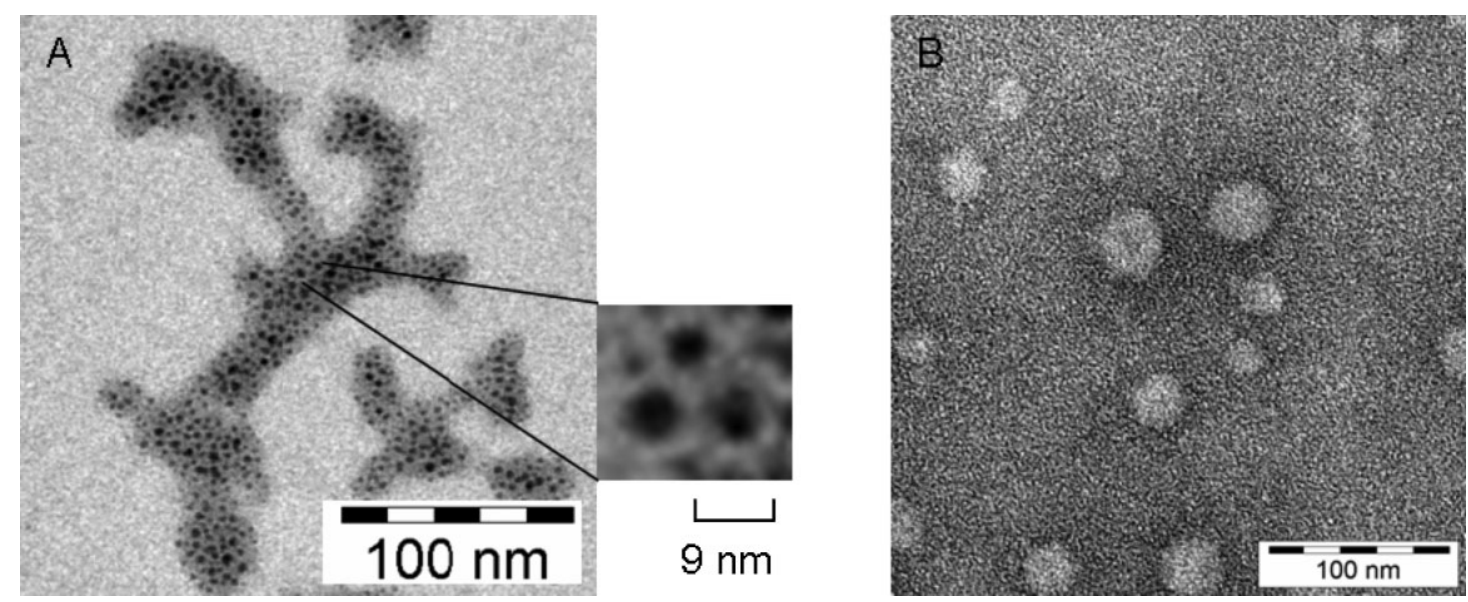

Fig. 5. TEM micrographs of (A) Hcp1-CdSe $4.5 \mathrm{~nm} Q D$ conjugates with the QD trapped inside of the yoctowell and (B) micrograph of Hcp1-9.5 $\mathrm{nm} Q D$ conjugates: the 9.5 $\mathrm{nm}$ QDs surrounded by Hcp1 donut forming a 30-40 nm multi-toroid shaped structure. 
not contain protein, nor showed any PL. The greatest PL intensity correlated with the highest protein concentration in the elution fractions for the Hcp1-9.5 nm QD conjugates (see Figure S4 and S5 in the Supporting Information). This clearly indicates the accessibility of the Hcp1 His-tags because $\mathrm{Ni}^{2+}$ ions still coordinate with the six histidine residues during the purification on the $\mathrm{Ni}^{2+}$-NTA column. (B) Fluorescence images of both Hcp1-4.5 QD and Hcp1-9.5 QD reveal a strong CdSe-signal (see Figure S7 in the Supporting Information). Both superseding structures look similar in fluorescence images due to unspecific particle interaction. But if TEM (Figure 5B) is applied a very different fine structure depending on the QD size used is observed. The recorded TEM micrographs clearly reveal very homogeneous spherical cluster of $30-40 \mathrm{~nm}$ for the Hcp1-CdSe $9.5 \mathrm{~nm}$ conjugates (Figure 5B). Hcp1 is very likely to bind the $9.5 \mathrm{~nm}$ QDs via the c-terminal His-tag facing outwards forming a multi-ring structure around the $9.5 \mathrm{~nm}$ QD (Figure 1B). The described multi-ring like structures can be deduced from the strong affinity of the six his tag to the QDs. The conjugation of proteins to metal nanoparticles as $\mathrm{Zn}^{2+}, \mathrm{Ni}^{2+}, \mathrm{Cu}^{2+}$ but also $\mathrm{Cd}^{2+}$ via the six histidine motive is a standard conjugation approach of peptide-QD binding. ${ }^{[11,22,24]}$ In this way, the QDs are completely shielded but also prone for aggregation. In contrast, the 2.5 and $4.5 \mathrm{~nm}$ QDs are incorporated in the middle of the donut and thus covalently attached to the six cysteine groups at the inside of the Hcp1 (Figure 5A and Figure S8 in the Supporting Information). (C) The formed hybrid structures are stable in aqueous solution for more than three months with a minor loss in PL intensity (see Figure S6 in the Supporting Information) This long-term stability highlights the steady interaction and protection of the QDs by Hcp1 rings. Not only the PL intensity of the QD is affected

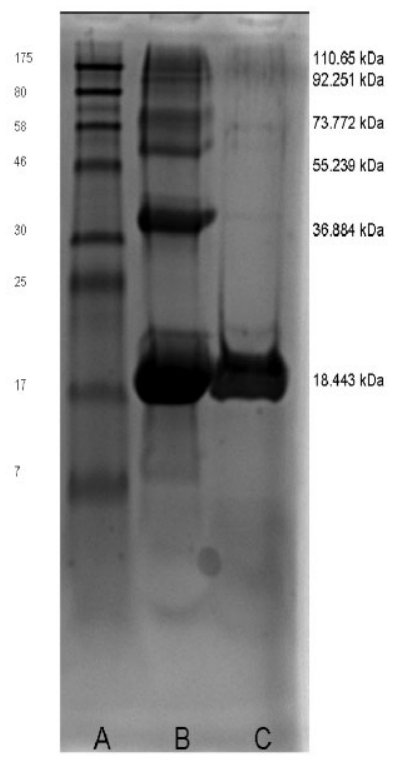

Fig. 6. SDS-PAGE of protein ladder (A) Hcp1-4.5 $\mathrm{nm} Q D$ conjugates (B) and Hcp1 native $(C)$. In lane $(B)$ monomers $(18.4 \mathrm{kDa})$, dimers $(36.9 \mathrm{kDa})$ up to hexamers $(110.7 \mathrm{kDa})$ of Hcp1-4.5 $\mathrm{nm}$ QD can be detected. by the conjugation but also the Hcp1 hexamer is stabilized through the 2.5-9.5 nm QD binding. In Figure 6, monomers, dimers, up to hexamers can be found even after boiling the Hcp1-QD hybrid structures for $5 \mathrm{~min}$ in 2\% SDS usually completely disintegrating quaternary protein interactions, which indicates a strong $4.5 \mathrm{~nm}$ QD-Hcp1 binding due to thiol-QD interactions. Nevertheless, if stirred for more than $1 \mathrm{~h}$ also unspecific binding via the His-tag, hydrophobic interactions or carboxy groups of Hcp1 might occur. Without applying denaturing conditions (boiling and SDS), the conjugates do not enter a native polyacrylamide gel $(\mathrm{PAG})^{[26]}$ (data not shown), which indicates that larger constructs of QDs and Hcp1 are broken down through boiling by preventing unspecific protein and Hcp1-QD interactions. To inhibit aggregation of the formed conjugates, milder reaction conditions for the phase transfer were applied by shaking the two solvent phases at $1400 \mathrm{rpm}$ for $2 \mathrm{~h}$ at $20^{\circ} \mathrm{C}$ (instead of stirring for $20 \mathrm{~h}$ ). Even though the recorded PL intensity of the bioconjugates is less (about five times less compared to the previous method), the buffer phase stays transparent and no evidence for macroscopic aggregation is observed. To proof specific thiol binding of the QDs under milder conditions the thiols inside the cavity of Hcp1 were blocked to suppress the QD-thiol interaction. Therefore, Hcp1 was reacted with maleimide via thio-Michael addition prior the QD-conjugation (see Supporting Information for experimental details). According to the results from trypsin digests and the Supporting Information at least $50 \%$ of the thiols are reacted with maleimide and thus blocked from QD binding. For 3.5 and $4.5 \mathrm{~nm}$ QDs a threefold decrease in PL intensity can be found for maleimide modified Hcp1conjugates (54C is partially blocked) compared to the unmodified proteins (Figure 7). This demonstrates the maleimide-induced inhibition of QD-thiol binding of the 3.5 and $4.5 \mathrm{~nm}$ QDs inside the protein cavity in the early reaction phase. As the transfer progresses the PL intensity difference of the modified and unmodified protein diminishes indicating successively binding via the His-tag or other interactions very likely associated with the formation of less specific aggregates. Only for the

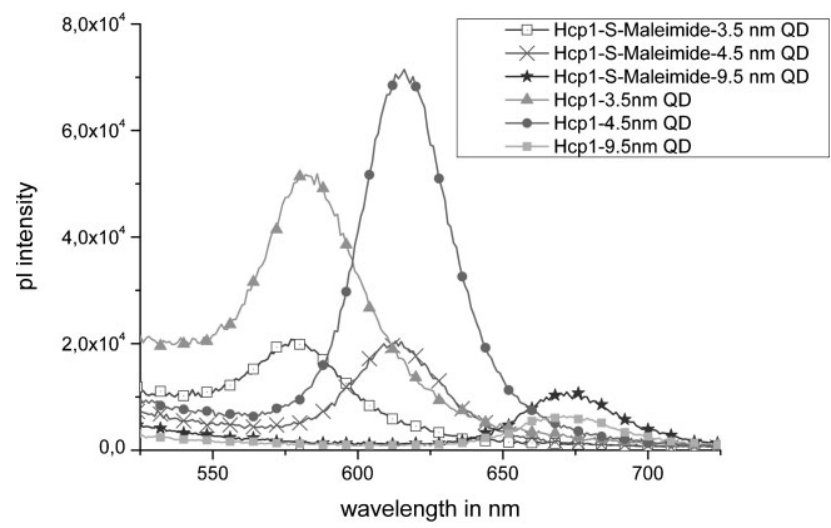

Fig. 7. PL intensity of Hcp1-QD in buffer with and without 54C-maleimide modification after shaking for $2 \mathrm{~h}$ at $1400 \mathrm{rpm}$ shows a decreased PL for Hcp1-maleimide modifications for 3.5 and $4.5 \mathrm{~nm}$ QDs but not for the $9.5 \mathrm{~nm}$ QD-conjugates. 
maleimide modified Hcp1, the $3.5 \mathrm{~nm}$ QD conjugates (see Figure S9 in the Supporting Information) a dominant differential PL intensity can be monitored. This suggests that the small sized $3.5 \mathrm{~nm}$ QDs precisely fit into the cavity and tend to bind less at the outside of Hcp1. In contrast, the $4.5 \mathrm{~nm}$ QDs, even though bound mainly inside the protein cavity $(4 \mathrm{~nm}$ width-static crystal structure geometry) slightly surmount the cavity and thus, are still accessible for further protein interactions. For the $9.5 \mathrm{~nm}$ QD-Hcp1 conjugates, no reduced PL intensity for the maleimide modified proteins can be monitored (Figure 7). Therefore, it is evident that binding of the $9.5 \mathrm{~nm}$ QDs does not occur inside the cavity but at the outside of the hexameric ring as shown earlier (Figure 5B). To further characterize the Hcp1-QD conjugates and notably the conformation of Hcp1, CD, and tryptophan fluorescence spectroscopy was applied. CD-spectroscopy was adopted to assess the secondary structure of the Hcp1-QD conjugates. The recorded signals (Figure S10A in the Supporting Information) of the $3.5,4.5$, and $9.5 \mathrm{~nm}$ Hcp1 conjugates demonstrate an unchanged secondary structure after binding in the early reaction stage ( $2 \mathrm{~h}$ shaking) compared to native plain Hcp1 (no QD bound) in previous measurements. ${ }^{[27]}$ The unchanged CD-signal is in congruence with the findings of Behrens et al. for the attachment of Pd-particles to the pore protein $\mathrm{Sp} 1{ }^{[28]}$ The investigation of the quaternary structure after QD binding, via intrinsic tryptophan fluorescence (see Figure S10B in the Supporting Information) and SDS-PAG (Figure 6), proofed the maintained native hexameric form of Hcp1 upon QD conjugation. It can be summarized that Hcp1 maintains its native secondary and quaternary structure after QD binding. The 4.5 and $3.5 \mathrm{~nm}$ QDs-Hcp1 conjugation in the early reaction stage occurs via multiple thiol-QD-surface interaction at the inside of the protein yoctowell, whereas the 9.5 $\mathrm{nm}$ QDs binds via the His-tag at the outside of the protein.

By changing the QD size, different structured bioconjugates are available. To expand the diversity of complex bio-nanoarchitectures, a new building block was introduced. Therefore, one cysteine was inserted at the lower rim (N159C) and one at the upper rim (G91C) of Hcp1Q54C to get the triple cysteine mutant Hcp1Q54C-G91C-N159C (Hcp1cys3) (see Figure 1C and for experimental details Supporting Information).

As reported in the literature, protein bio-nanoarchitectures have been used to control specific self-assembly processes for various applications from conducting nanowires to the constrained synthesis and organization of catalytically active metal nanoparticles. ${ }^{[28-30]}$ Investigating the structure forming potential of the new Hcp1 mutant (Hcp1Cys3), $9.5 \mathrm{~nm}$ QDs (in DMSO) are added in presence of a reductive agent to a diluted Hcp1cys3 buffer solution. Fluorescence imaging reveals similar superseding structures as for Hcp1Q54C-QD conjugates due to unspecific particle interaction (Figure S7 in the Supporting Information). But the fine structures observed by TEM (Figure 8) for the Hcp1Cys3-9.5 nm QD conjugates are very different. The strong cysteine-QD linkage leads to the self-assembly of photonic wires. Those wires cannot be

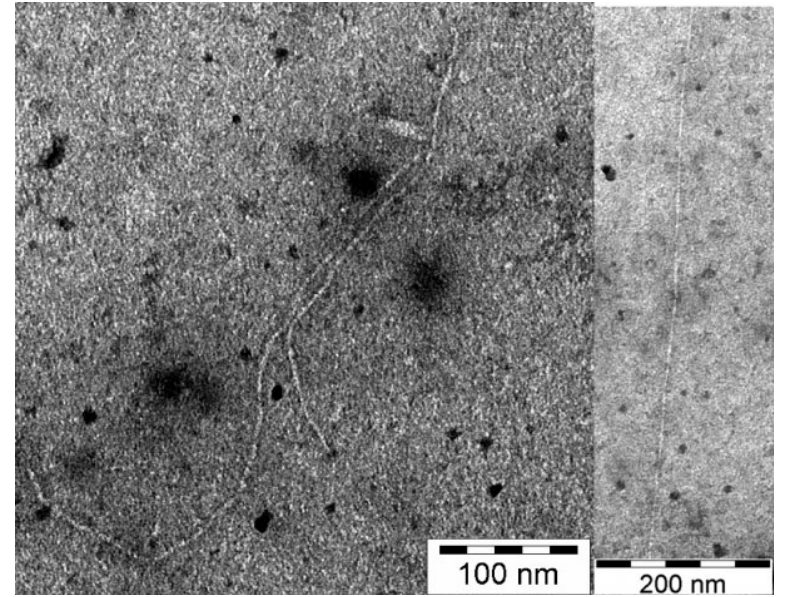

Fig. 8. TEM micrographs: Hcp1cys3 conjugated via 9.5 QDs forming tubes of about $9 \mathrm{~nm}$ widths and up to $850 \mathrm{~nm}$ length, negatively stained with uranyl formate (black dots).

detected for the Hcp1cys3 mutant with 4.5 and $3.5 \mathrm{~nm}$ QD conjugates nor for Hcp1cys3 alone under same reductive conditions. To generalize this approach also CdTe $8.5 \mathrm{~nm}$ QDs with Hcp1cys3 self-assembled into wired structures (Figure S11 in the Supporting Information).

\section{Conclusions}

In the work presented here, we show, to the best of our knowledge, for the first time the size dependent conjugation of differently sized CdSe QDs with defined protein architectures, in our case the donut-shaped protein (Hcp1). The CdSe QDs encapsulated by Hcp1 protein yoctowells are highly stable resulting in PL conservation for more than 3 month. Solvent type, QD size and ligand chemistry strongly influence the bio-conjugation, the resulting molecular assemblies and the PL intensity of the hybrid nanoarchitectures. After adjusting the reaction conditions (shaking with PBS buffer/toluene or DMSO and pyridine as QD ligand), major general requirements for bioconjugation are fulfilled. The PL intensity of the conjugated QD is conserved as well as the native structure of the bound Hcp1 protein hexamers. Furthermore, 2.5, 3.5, and $4.5 \mathrm{~nm}$ QDs form molecularly defined conjugates given that only one hexamer is linked to each QD.

Thus, defined nanostructures could be assembled from single QDs trapped inside the yoctowell over multi-toroid-like architectures to photonic wires with up to $850 \mathrm{~nm}$ in length and $9 \mathrm{~nm}$ in width. Future research will include Hcp1Q54C functionalization with epitopes used for specific stoichiometric (one QD-one protein hexamer composed of six ligands) labeling and targeting of cell compartments in vivo. As Hcp1 is related to the infection mechanism of cystic fibrosis, ${ }^{[14]}$ it potentially has a positive effect on the uptake of QDs across the cell membrane. Further applications include the utilization of such photonic nanosystems, selectively modified via bisfunctional linkers, for the assembly of defined 
bio-based photo-energy converting architectures mimicking biological systems.

[1] T. Pellegrino, L. Manna, S. Kudera, T. Liedl, D. Koktysh, A. L. Rogach, S. Keller, J. Rädler, G. Natile, W. J. Parak, Nano Lett. 2004, 4, 703.

[2] I. Willner, F. Patolsky, J. Wasserman, Angew. Chem., Int. Ed. 2001, 40, 1861

[3] T. Torimoto, M. Yamashita, S. Kuwabata, T. Sakata, H. Mori, H. Yoneyama, J. Phys. Chem. B 1999, 103, 8799.

[4] H. Mattousi, J. M. Mauro, E. R. Goldman, G. P. Anderson, V. C. Sundar, F. V. Mikulec, M. G. Bawendi, J. Am. Chem. Soc. 2000, 122, 12142.

[5] J. P. a. T. P. a. C. P. Wolfgang, Nanotechnology 2005, 16, R9.

[6] L. Wang, D. Nagesha, S. Selvarasah, M. Dokmeci, R. Carrier, J. Nanobiotechnol. 2008, 6, 11.

[7] A. M. Derfus, W. C. W. Chan, S. N. Bhatia, Nano Lett. 2003, 4, 11 .

[8] A. Fahmi, T. Pietsch, M. Bryszewska, J. C. RodríguezCabello, A. Koceva-Chyla, F. J. Arias, M. A. Rodrigo, N. Gindy, Adv. Funct. Mater. 2010, 20, 1011.

[9] I. L. Medintz, H. T. Uyeda, E. R. Goldman, H. Mattoussi, Nat. Mater. 2005, 4, 435.

[10] P. K. Chattopadhyay, S. P. Perfetto, J. Yu, M. Roederer, Wiley Interdisciplinary Reviews: Nanomed. Nanobiotechnol. 2010, 2, 334.

[11] I. L. Medintz, J. H. Konnert, A. R. Clapp, I. Stanish, M. E. Twigg, H. Mattoussi, J. M. Mauro, J. R. Deschamps, Proc. Natl. Acad. Sci. U. S. A. 2004, 101, 9612.

[12] R. Majithia, J. Patterson, S. E. Bondos, K. E. Meissner, Biomacromolecules 2011, 12, 3629.

[13] R. P. Bagwe, X. Zhao, W. Tan, Bioconjugated Luminescent Nanoparticles for Biological Applications, Taylor \& Francis, Philadelphia, PA ETATS-UNIS 2003.
[14] J. D. Mougous, M. E. Cuff, S. Raunser, A. Shen, M. Zhou, C. A. Gifford, A. L. Goodman, G. Joachimiak, C. L. Ordonez, S. Lory, T. Walz, A. Joachimiak, J. J. Mekalanos, Science 2006, 312, 1526.

[15] Y. Yuan, F.-S. Riehle, H. Gu, R. Thomann, G. Urban, Kr. M. Ger, J. Nanosci. Nanotechnol. 2010, 10, 6041.

[16] W. W. Yu, L. Qu, W. Guo, X. Peng, Chem. Mater. 2003, 15, 2854.

[17] M. E. Ãkerman, W. C. W. Chan, P. Laakkonen, S. N. Bhatia, E. Ruoslahti, Proc. Natl. Acad. Sci. 2002, 99, 12617.

[18] D. M. Willard, L. L. Carillo, J. Jung, A. Van Orden, Nano Lett. 2001, 1, 469.

[19] G. Chornokur, et al., Semicond. Sci. Technol. 2008, 23, 075045.

[20] R. W. Meulenberg, T. Jennings, G. F. Strouse, Phys. Rev. B 2004, 70, 235311.

[21] F. Pinaud, D. King, H.-P. Moore, S. Weiss, J. Am. Chem. Soc. 2004, 126, 6115.

[22] J. M. Slocik, J. T. Moore, D. W. Wright, Nano Lett. 2002, 2, 169.

[23] J. F. Hainfeld, W. Liu, C. M. R. Halsey, P. Freimuth, R. D. Powell, J. Struct. Biol. 1999, 127, 185.

[24] I. L. Medintz, M. H. Stewart, S. A. Trammell, K. Susumu, J. B. Delehanty, B. C. Mei, J. S. Melinger, J. B. BlancoCanosa, P. E. Dawson, H. Mattoussi, Nat Mater. 2010, 9, 676.

[25] D. Dorokhin, N. Tomczak, A. H. Velders, D. N. Reinhoudt, G. J. Vancso, J. Phys. Chem. C 2009, 113, 18676.

[26] C. Dockter, A. Volkov, C. Bauer, Y. Polyhach, Z. Joly-Lopez, G. Jeschke, H. Paulsen, Proc. Natl. Acad. Sci. U. S. A. 2009, 106, 18485.

[27] A. Schreiber, E. Zaitseva, Y. Thomann, R. Thomann, J. Dengjel, R. Hanselmann, S. M. Schiller, Soft Matter 2011, 7, 2875.

[28] S. Behrens, A. Heyman, R. Maul, S. Essig, S. Steigerwald, A. Quintilla, W. Wenzel, J. Bürck, O. Dgany, O. Shoseyov, Adv. Mater. 2009, 21, 3515.

[29] M. T. Kumara, B. C. Tripp, S. Muralidharan, Chem. Mater. 2007, 19, 2056

[30] T. Scheibel, R. Parthasarathy, G. Sawicki, X.-M. Lin, H. Jaeger, S. L. Lindquist, Proc. Natl. Acad. Sci. 2003, $100,4527$. 